\title{
Cooling of a Strange Star with Crust
}

\author{
Pierre M. Pizzochero \\ Physics Department, State University of New York at Stony Brook, Stony Brook, New York 11794 \\ and Istituto Nazionale di Fisica Nucleare, Sezione di Milano, Via Celoria 16, I-20133 Milano, Italy \\ (Received 15 October 1990)
}

\begin{abstract}
The cooling history of a strange star surrounded by a thin crust of ordinary matter is followed by solving analytically the general-relativistic equation of heat transfer across the crust. The surface temperature of the strange star is found to be lower than that of a neutron star (ordinary or with core condensates) of the same age. This provides a definite observational signature for these exotic objects, so far the first quantitative one to be proposed.
\end{abstract}

PACS numbers: $97.60 . J d, 12.38 . \mathrm{Mh}, 97.10 . \mathrm{Ri}$

Strange stars have been proposed as a new class of astronomical compact objects, and various physical scenarios have been proposed and discussed theoretically. ${ }^{1-3}$ In order to confirm the existence of such stars, some characteristic signature must be found and compared to observations.

We consider here a star consisting of a core of strange quark matter surrounded by a thin crust of ordinary matter. ${ }^{3}$ We point out that this is not the only possible scenario, although a quite plausible one, and the details about its formation and stability are still far from being understood. In this Letter we show that the high cooling rate of quark matter together with the quick thermal response of the thin crust yield low surface temperatures as compared to neutron stars of the same age. This provides a definite observational signature for this scenario, so far the first one to be proposed in quantitative terms.

After the formation of the star, the core cools very rapidly due to intense neutrino emission and the temperature at the surface depends on the transfer of heat across the crust. Our approach relies on two main assumptions: (i) the pressure in the crust is dominated by the contribution from degenerate relativistic electrons, and (ii) the thermal behavior of the crust is that of a simple solid, i.e., its specific heat $C$ is that of a classical crystal and its thermal conductivity $\kappa$ is given by the contribution from electron-phonon scattering in the ion lattice.

Under these assumptions the density structure of the crust can be solved analytically and the generalrelativistic heat equation can be integrated exactly to give the temperature response of the surface to the cooling of the core. The first approximation turns out to be a very good one over most of the crust, with the exception of the thin "skin" of nondegenerate matter whose effect on the surface temperature will be treated separately at the end. The second assumption is a more coarse approximation, since the outer part of the crust (about one-third of its total extension) is in the liquid phase. However, we believe that this does not significantly affect our final results and general conclusions, as we will discuss later.
The structure of the crust is determined by the general-relativistic equation of hydrostatic equilibrium. The crust is thin $\left(\sim \frac{1}{2} \mathrm{~km}\right)$ and contains negligible mass $\left(<10^{-4} M_{\odot}\right)$ so that the equation simplifies to $d P / d z$ $=\rho g_{s}$, where $g_{s}$ is the surface gravity and $z$ is the general-relativistic depth coordinate. ${ }^{4}$ If $M$ and $R$ are the total mass and the radius of the star, and $r$ is the radial coordinate, we have $g_{s}=e^{-\phi} G M / R^{2}$ and $z=e^{-\phi}(R$ $-r$ ), with $e^{2 \phi}=1-2 G M / R c^{2}$. The total mass density $\rho$ is practically equal to the rest mass density of the nonrelativistic ions in the lattice, since the contribution of the electrons to the total energy can be neglected. From the assumption that the pressure comes entirely from the degenerate relativistic electrons we have $P=1.25 \times 10^{15}$ $\times \xi\left(Y_{e} \rho\right)^{4 / 3}$. Here $Y_{e}=Z / A$ is the electron fraction for ions with atomic number $Z$ and mass number $A$, while $\xi=1-0.0046 Z^{2 / 3}$ is the Coulomb correction to the pressure from the lattice; ${ }^{5}$ we use cgs units throughout. Finally, the density profile for the crust in the degenerate regime is given by $\rho=8 \times 10^{-48}\left(g_{s} / \xi\right)^{3} Y_{e}^{-4} z^{3}$. The crust extends down to a depth $z_{c}$ and density $\rho_{c}$ where it is in contact with the inner quark core. Stability arguments for the conversion of ordinary matter into strange quark matter show that the maximum crust which can be supported by a strange star corresponds to $\rho_{c}=\rho_{\text {nd }}$, where $\rho_{\text {nd }}=4 \times 10^{11} \mathrm{~g} / \mathrm{cm}^{3}$ is the neutron drip density. ${ }^{3}$

The core cools rapidly due to intense neutrino emission from the $\beta$ decay of degenerate relativistic quarks. The neutrino emissivity $\epsilon_{v}$ depends on the mass $m_{s}$ of the strange quark and on the QCD coupling constant $\alpha_{c}$ $=g^{2} / 4 \pi$, and so far in the literature there is no agreement on its expression. ${ }^{3,6}$ Here we will take $\epsilon_{v}=9$ $\times 10^{-30} T^{6} \mathrm{erg} / \mathrm{cm}^{3} \mathrm{~s}$ as obtained from Ref. 6 for $m_{s}$ $=200 \mathrm{MeV}$ and $\alpha_{c}=0.4$; this should give at least the correct order of magnitude. Assuming that the density is roughly constant, which for the self-bound core is a reasonable approximation, and taking a uniform initial temperature $T_{i}$, the temperature of the core at time $t$ is then given by $T_{c}(t)=T_{i}\left(1+\epsilon T_{i}^{4} t\right)^{-1 / 4}$, where $\epsilon=1.7$ $\times 10^{-30} R^{2} M^{-2 / 3}$.

Because of the rapidity of the cooling and of the difference in their masses, the core acts on the crust as a 
heat reservoir. The thermal behavior of the latter can therefore be found by solving the heat transfer problem with $T_{c}(t)$ as a boundary condition and neglecting the less intense neutrino emission which takes place in ordinary matter. The general-relativistic heat equation for a thin crust without sinks or sources of energy is ${ }^{4}$

$$
C \partial T(z, s) / \partial s=(\partial / \partial z)[\kappa \partial T(z, s) / \partial z]
$$

where $s=e^{\phi} t$ is the proper time and $T(z, s)$ is the temperature of the crust at relativistic depth $z$ and at proper time $s$. The equipartition theorem gives for the specific heat of a classical lattice of ions with number density $N_{Z}$ the expression $C=3 k N_{Z}=2.5 \times 10^{8} A^{-1} \rho$. For the solid crust this is a reasonable approximation since quantum corrections of the Debye model are negligible. The thermal conductivity from electron-phonon scattering is a function of the electron chemical potential, and for degenerate relativistic electrons is given by $\kappa=3.3 \times 10^{11}$ $\times\left(Y_{e} \rho\right)^{2 / 3}{ }^{7}$ From our density profile we can write $C=a_{1} z^{3}$ and $\kappa=a_{2} z^{2}$, where $a_{1}$ and $a_{2}$ are constants depending on $Z, A$, and $g_{s}$.

We define dimensionless variables $x=z / z_{c}$ and $\tau=s$ / $s_{c}$, where

$$
s_{c}=\left(a_{1} / a_{2}\right) z_{c}^{3}=1.9 \times 10^{28}\left(\xi Y_{e} / g_{s}\right)^{2} A^{-1} \rho_{c} .
$$

We also define dimensionless functions $\Theta=1-T / T_{i}$ and $F=1-T_{c} / T_{i}$; in particular, we have

$$
F(\tau)=1-(1+a \tau)^{-1 / 4}
$$

with the dimensionless parameter $a=\epsilon T_{i}^{4} s_{c}$. The heat equation is then

$$
\partial \Theta(x, \tau) / \partial \tau=x^{-3}(\partial / \partial x)\left[x^{2} \partial \Theta(x, \tau) / \partial x\right] .
$$

The boundary and initial conditions for the function $\Theta(x, \tau)$ are $\Theta(1, \tau)=F(\tau), \Theta(0, \tau)<\infty$, and $\Theta(x, 0)=0$, where we have required that the temperature at the boundary $z=z_{c}$ is equal to the core temperature, that the temperature at the surface is finite, and that the initial temperature of the crust has also the constant value $T_{i}$.

Equation (1) can be integrated exactly by separation of variables and using Duhamel's theorem for timedependent boundary conditions. The solution is in terms of Bessel functions of order one-third, and it can be written as $\Theta(x, \tau)=F(\tau) \mathcal{R}(x, \tau)$, where we introduce the response function

$$
\begin{aligned}
\mathcal{R}(x, \tau)=1-\sum_{n=1}^{\infty} & 3\left[\lambda_{n} J_{4 / 3}\left(\frac{2}{3} \lambda_{n}\right)\right]^{-1} \\
& \times x^{-1 / 2} J_{1 / 3}\left(\frac{2}{3} \lambda_{n} x^{3 / 2}\right) I_{n}(\tau) / F(\tau) .
\end{aligned}
$$

Here $\frac{2}{3} \lambda_{n}$ are the eigenvalues of the Bessel function, i.e., $J_{1 / 3}\left(\frac{2}{3} \lambda_{n}\right)=0$. The functions $I_{n}$ are defined by the following integral representation:

$$
I_{n}(\tau)=(a / 4) \int_{0}^{\tau} e^{-\lambda_{n}^{2} \tau^{\prime}}\left[1+a\left(\tau-\tau^{\prime}\right)\right]^{-5 / 4} d \tau^{\prime}
$$

The temperature of the crust is simply related to its initial temperature and to the temperature of the core through the response function, i.e.,

$$
T(x, \tau)=[1-\mathcal{R}(x, \tau)] T_{i}+\mathcal{R}(x, \tau) T_{c}(\tau) .
$$

We notice the expected properties: $\mathscr{R}(x, \tau) \rightarrow 0$ as $\tau$ $\rightarrow 0, \mathcal{R}(x, \tau) \rightarrow 1$ as $\tau \rightarrow \infty$, and $\mathcal{R}(1, \tau)=1$. From the behavior of the Bessel functions for small values of their argument we also find that the response function does not depend on $x$ when $x \ll 1$, i.e., close to the surface it has the asymptotic expression

$\mathcal{R}_{0}(\tau)=1-\sum_{n=1}^{\infty}\left(3 / \lambda_{n}\right)^{2 / 3}\left[\Gamma\left(\frac{4}{3}\right) J_{4 / 3}\left(\frac{2}{3} \lambda_{n}\right)\right]^{-1} I_{n}(\tau) / F(\tau)$.

The transition of the electrons from the degenerate to the nondegenerate regime occurs roughly at $\rho \sim 10^{6}-10^{7}$ $\mathrm{g} / \mathrm{cm}^{3}$ corresponding to $x \sim 0.02$. Therefore the temperature at the outer boundary of the degenerate crust can be taken as

$$
T_{0}(\tau)=\left[1-\mathcal{R}_{0}(\tau)\right] T_{i}+\mathcal{R}_{0}(\tau) T_{c}(\tau) .
$$

The nondegenerate crust is very thin $(\sim 10 \mathrm{~m})$ and its thermal conductivity is dominated by photon transport. ${ }^{7}$ When compared to the cooling time scale of the degenerate crust the characteristic time of thermal response of this skin turns out to be very short, of the order of 1 day as results from simple random-walk arguments. Therefore, we can assume that the temperature $T_{s}(\tau)$ at the surface of the star follows immediately the variation with time of the temperature $T_{0}(\tau)$ at the boundary between the degenerate and nondegenerate region. The relation between their magnitudes has been studied both numerically and analytically; we will use the results of Ref. 7 where the following expression is derived for the drop in temperature across a skin of iron atoms: $T_{s}(\tau)=1.05$ $\times 10^{-3} g_{s}^{1 / 4} T_{0}(\tau)^{m}$, with $m=0.708$. Finally, the gravitationally redshifted surface temperature as measured by a distant observer at time $t$ is given by $T_{s}^{\infty}(t)=e^{\phi} T_{s}(\tau$ $\left.=e^{\phi} t / s_{c}\right)$.

Let us now discuss the physical implications of our calculation. The crust acts as an insulating layer that slows down the thermal signal from the rapidly cooling core. The efficiency of this delay along the crust and with time is expressed by the response function $R(x, \tau)$ : A zero value indicates perfect insulation from the core, while a unit value indicates that thermal equilibration with the core has been reached. The response function depends on the parameter $a=\epsilon T_{i}^{4} s_{c}$. However, for realistic situations one finds that $a \sim 10^{4}-10^{8}$ and in this range the response function turns out to be practically independent from $a$ provided that $\tau>0.05$. Since $s_{c} \sim 5-10 \mathrm{yr}$, this corresponds to a time of a few months after the strange star has formed. The behavior of $\mathcal{R}(x, \tau)$ under these conditions is plotted in Fig. 1 which clearly shows the propagation with time of the thermal signal from the inner edge $(x=1)$ to the outer edge 


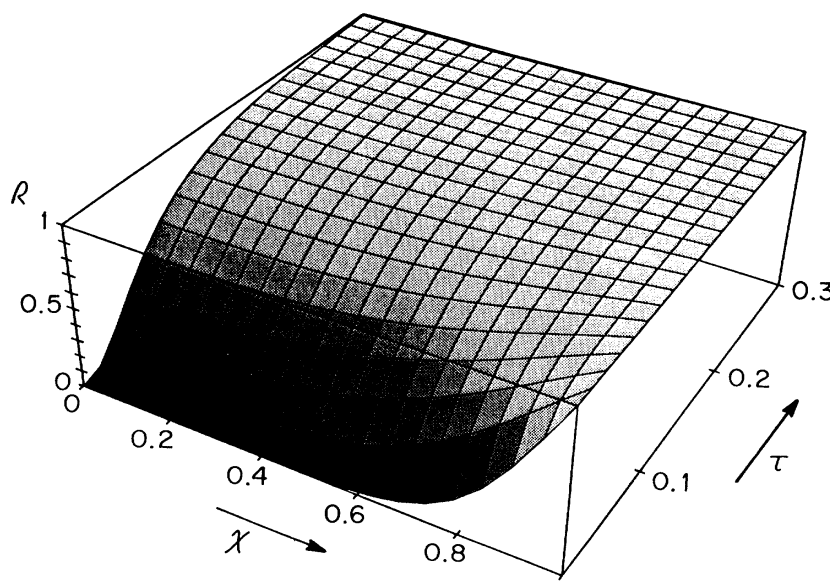

FIG. 1. Response function $\mathscr{R}(x, \tau)$ calculated for a typical value of the parameter $a=10^{6}$. The dimensionless axis variables are $x=z / z_{c}$ and $\tau=s / s_{c}$, with $0 \leq x \leq 1$ and $0.01 \leq \tau$ $\leq 0.3$.

$(x=0)$ of the crust. In particular, we see that after $\tau \simeq 0.3$ the response function is unity across the entire crust, i.e., it takes only a few years for the surface of the star to equilibrate with its cold core. This is in sharp contrast to the case of neutron stars, with or without core condensates, where the thicker crusts give equilibration times of the order of several tens of years. ${ }^{8,9}$ This physical difference provides a characteristic signature to distinguish strange stars from neutron stars.

We choose the following realistic parameters, as suggested both by observations and by theoretical modeling of compact objects: $M=1.4 M_{\odot}, R=10-12 \mathrm{~km}, Z=28$, and $A=84 .{ }^{10}$ We also take $\rho_{c}=\rho_{\text {nd }}$, i.e., we consider the maximum allowed crust; thinner crusts are less insulating and give a faster cooling of the surface during the first few years. Finally, we consider initial temperatures in the range $T_{i} \sim 10^{9}-10^{10} \mathrm{~K}$, which is typical for compact objects formed in stellar collapse and observed in supernova remnants. In Table I we summarize the physical parameters for the cases considered.

We notice that the thermal diffusivities $D=\kappa / C$ calculated for a solid and a liquid differ by less than a factor of 2 in the range of interest. We have checked that scaling $D$ by this amount does not affect the values obtained

TABLE I. Physical parameters calculated for a strange star with crust assuming that $M=1.4 M \odot, Z=28, A=84$, and $\rho_{c}=\rho_{\text {nd. }}$.

\begin{tabular}{lcc}
\hline \hline & $R=10 \mathrm{~km}$ & $R=12 \mathrm{~km}$ \\
\hline$e^{\phi}$ & 0.77 & 0.81 \\
$g_{s}(\mathrm{dyn} / \mathrm{g})$ & $2.4 \times 10^{14}$ & $1.6 \times 10^{14}$ \\
$z_{c}(\mathrm{~m})$ & 334 & 509 \\
$s_{c}(\mathrm{yr})$ & 4.9 & 11.4 \\
$a T_{i}^{-4}\left(\mathrm{~K}^{-4}\right)$ & $1.3 \times 10^{-32}$ & $4.4 \times 10^{-32}$ \\
\hline \hline
\end{tabular}

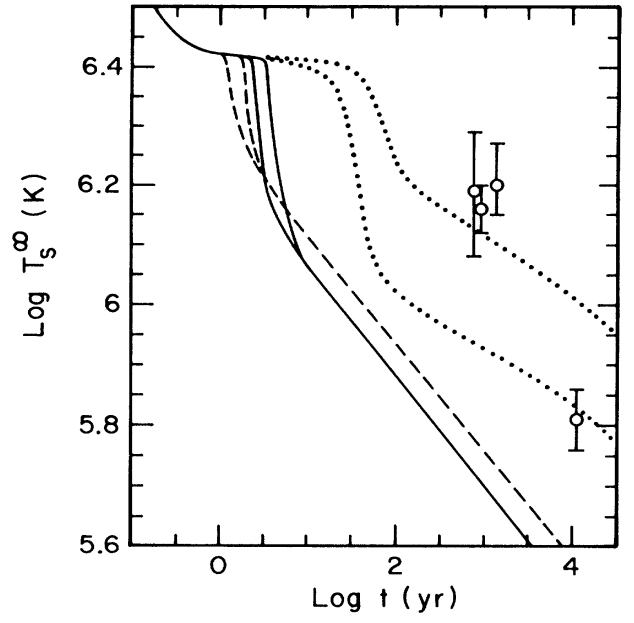

FIG. 2. Surface temperature $T_{s}^{\infty}(t)$ as measured by a distant observer. The results for a strange star corresponding to the parameters in Table I are given by the dashed lines $(R=10$ $\mathrm{km})$ and the solid lines $(R=12 \mathrm{~km})$, the upper line for each case corresponding to $T_{i}=10^{10} \mathrm{~K}$ and the lower one to $T_{i}=10^{9}$ K. The dotted lines are the results of Ref. 9 for an ordinary neutron star (upper line) and for a neutron star with strangeness condensation and nucleon superfluidity (lower line). The points are the observations of the Einstein Observatory, the compact sources being from left to right 3C 58, Crab, RC 103, and Vela.

for $T_{s}^{\infty}$ after a few years, and this justifies our initial approximations in describing the crust.

In Fig. 2 we show the results of our cooling calculation for the redshifted surface temperatures $T_{s}^{\infty}(t)$. Detailed calculations have shown that surface emission from the skin quickly brings the temperature down to $T_{s}^{\infty} \simeq 2.6$ $\times 10^{6} \mathrm{~K},{ }^{11}$ so that we plot our results only after they fall below this value. In addition to the curves corresponding to the different parameters discussed above, we also give the results for the cooling of an ordinary neutron star and of a neutron star with effects of strangeness (kaon) condensation and nucleon superfluidity taken into account, as obtained for a $1.4 M_{\odot}$ star in the recent work by Page and Baron (cf. Ref. 9 for details). The result for the latter case is not unique since the critical parameters for such phase transitions are not known, but it corresponds to a good fit to the low temperature measured for the Vela pulsar. Finally, the points represent estimated surface temperatures of four sources obtained from the Einstein Observatory data assuming $M=1.4 M_{\odot}$ and $R=10 \mathrm{~km} .{ }^{12}$ We have only reported those observations which correspond to the actual detection of a compact object. Actually, these temperatures should be viewed only as upper limits to $T_{s}^{\infty}$.

From the results in Fig. 2 we see that the surface temperature of a strange star with crust is lower than that of a neutron star (ordinary or with a condensate in the core) of the same age. The same general behavior is 
found for different parameters and thus this provides a definite signature for the identification of a strange star. In particular, the prompt drop in temperature at early times (between about 5 and $50 \mathrm{yr}$ since formation) is due to the small thermal equilibration time of the thin crust, while the low temperatures reached at later times are due to fast cooling of the core. Therefore, taking different condensates in the center of a neutron star with neutrino emissivities higher than in the kaon case (e.g., pion condensate or quark matter in the core) will decrease the temperature difference between a strange and a neutron star at later times (not wipe it out though, since the rapid cooling interests the whole mass of the strange star but only the core of the neutron star), while it will not affect the early behavior. This guarantees that the (early) surface temperature is actually a good observational signature and it stresses the relevance of future observations of young compact objects.

We could now compare the results of our calculation for the cooling of a strange star with crust to the observations of known compact sources, in order to decide on observational grounds whether presently there is any evidence (positive or negative) for the identification of these exotic astronomical objects. Unfortunately, the fact that the astronomical measurements provide only upper limits on $T_{s}^{\infty}$ does not allow one to draw any firm conclusion. However, if we tentatively assume that the actual surface temperatures are close to their upper limits, as always done in the literature, we see from Fig. 2 that none of the presently known compact sources is compatible with the cooling pattern of a strange star with crust, while their thermal behavior is well described by the neutron-star scenario (cf. Ref. 9). This negative evidence would not rule out the existence of older $\left(>10^{4}\right.$ yr) strange stars, formed from the conversion of very old neutron stars. In fact, the mechanism of formation of strange stars is a totally open issue ${ }^{3}$ and observations could actually set a lower limit on the rate of conversion to strange quark matter.

Strange stars have been widely discussed, particularly after the erroneous observation of a $\frac{1}{2}-\mathrm{ms}$ pulsar in $\mathrm{SN}$
1987A. The issue of their existence is both a nuclearphysics problem and an astrophysics one. The former is concerned with the stability of strange quark matter, and the latter with some astronomical evidence for these new stars. In this Letter we have shown that the cooling of the surface of a strange star with crust, particularly between 5 and $50 \mathrm{yr}$ since formation, offers a good signature of these objects, so far the only one to be proposed. Future extended observations of (young) compact objects - the remnant in SN 1987A being the first candidate, after the absorption from the circumstellar material has decreased sufficiently-will provide us with new experimental information.

The author wishes to thank Dany Page for useful discussions. This work was supported by the U.S. Department of Energy under Contract No. DE-FG02-88ER40388.

${ }^{1}$ E. Witten, Phys. Rev. D 30, 272 (1984).

${ }^{2}$ P. Haensel, J. L. Zdunik, and R. Schaeffer, Astron. Astrophys. 160, 121 (1986).

${ }^{3}$ C. Alcock, E. Farhi, and A. Olinto, Astrophys. J. 310, 261 (1986).

${ }^{4}$ E. Gudmundsson, C. Pethick, and R. Epstein, Astrophys. J. 272, 286 (1983).

${ }^{5}$ S. L. Shapiro and S. A. Teukolsky, Black Holes, White Dwarfs and Neutron Stars (Wiley, New York, 1983).

${ }^{6}$ R. C. Duncan, S. L. Shapiro, and I. Wasserman, Astrophys. J. 267, 358 (1983).

${ }^{7}$ L. Hernquist and J. H. Applegate, Astrophys. J. 287, 244 (1984).

${ }^{8}$ G. E. Brown, K. Kubodera, D. Page, and P. Pizzochero, Phys. Rev. D 37, 2042 (1988).

${ }^{9}$ D. Page and E. Baron, Astrophys. J. Lett. Ed. 354, L17 (1990).

${ }^{10} \mathrm{P}$. Hansel, J. L. Zdunik, and J. Dobaczewsky, Astron. Astrophys. 222, 353 (1989).

${ }^{11}$ D. Page, Ph.D. thesis, State University of New York at Stony Brook, 1989 (unpublished).

${ }^{12} \mathrm{~S}$. Tsuruta, Comments Astrophys. 11, 151 (1986). 